\title{
Helioseismic probing of the subsurface structure of sunspots
}

\author{
A.D. Crouch, A.C. Birch, D.C. Braun, and C.T.M. Clack
}

NorthWest Research Associates, Colorado Research Associates Division 3380 Mitchell Lane, Boulder, CO

\begin{abstract}
We discuss recent progress in the helioseismic probing of the subsurface structure of solar magnetic regions. To simulate the interaction of helioseismic waves with magnetic fields and thermal perturbations we use a simple model that is translation invariant in the horizontal directions, has a realistic stratification in the vertical direction, and has physically consistent boundary conditions for the waves at the upper and lower boundaries of the computational domain. Using this model we generate synthetic helioseismic data and subsequently measure time-distance travel times. We evaluate a model for the wave-speed perturbation below sunspots that replaces the sound speed in a non-magnetic model by the fast-mode speed from a magnetic model; our results indicate that this approach is unlikely to be useful in modeling wave-speed perturbations in sunspots. We develop and test an inversion algorithm for inferring the soundspeed perturbation in magnetic regions. We show that this algorithm retrieves the correct soundspeed perturbation only when the sensitivity kernels employed account for the effects of the magnetic field on the waves and the subsurface structure.
\end{abstract}

Keywords. Sun: helioseismology, Sun: magnetic fields, sunspots

\section{Introduction}

One goal of local helioseismology is to infer the subsurface structure of sunspots and magnetic regions. At present there are two different general classes of helioseismic models for the wave-speed perturbation in sunspots (e.g., Gizon, Birch, \& Spruit, 2010). On the one hand, a shallow, positive wave-speed perturbation is favored by Fan, Braun, \& Chou (1995), Crouch et al.(2005), and Cameron et al.(2010). On the other hand, standard inversions from time-distance measurements favor a two-layer model with a negative wave-speed perturbation near the surface and a positive wave-speed perturbation in deeper layers (e.g., Kosovichev, Duvall, \& Scherrer, 2000, Couvidat et al., 2005, Hughes, Rajaguru, \& Thompson, 2005). One possible source of uncertainty is the effect of the magnetic field on both solar oscillations and structure. The aim of this investigation is to evaluate how magnetic fields may affect helioseismic inversion methods.

\section{Translation-invariant model for wave propagation (TIM)}

The translation-invariant model for wave propagation (TIM) solves the linearized MHD equations in a horizontally uniform, three-layer background model that may be permeated by a uniform, inclined magnetic field. The upper layer is an isothermal slab that simulates the influence of the solar atmosphere. The bottom layer is a polytrope that represents the deep solar interior. In the polytrope and separately in the isothermal slab semi-analytic solutions for the linearized MHD equations are developed (e.g., Crouch \& Cally, 2003, Crouch et al. 2005, Cally \& Goossens, 2008). Physical boundary conditions 

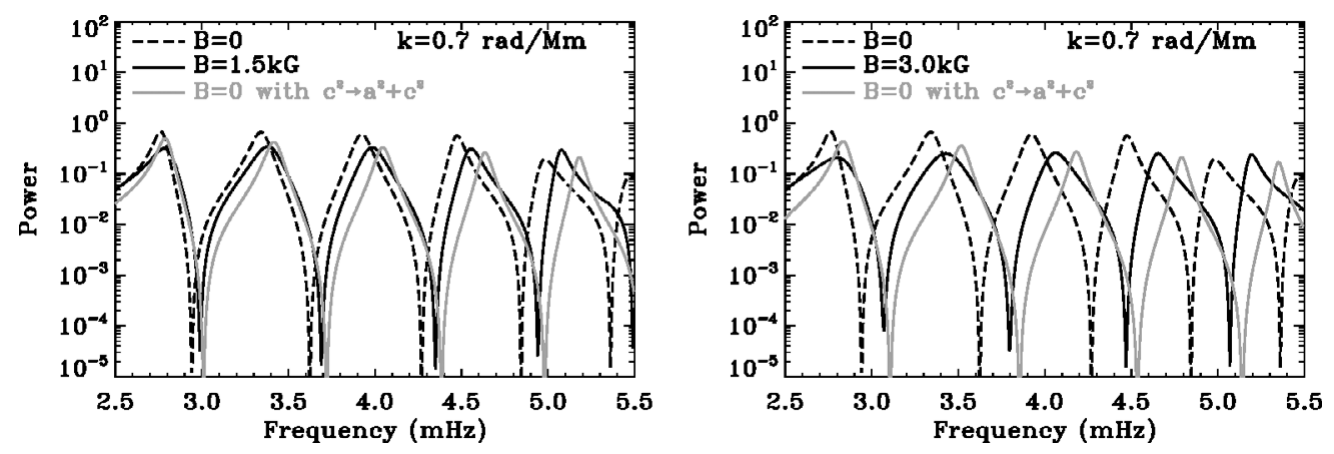

Figure 1. Power as a function of frequency at fixed horizontal wavenumber. In each panel the dashed curve is for a non-magnetic model and the solid black curve is for a model with a vertical magnetic field (left: $B=1.5 \mathrm{kG}$, right: $B=3 \mathrm{kG}$ ). A vertical field affects the power spectra in two ways: the ridges are shifted to higher frequency / horizontal phase speed and they have a larger linewidth (due to the additional damping from mode conversion to waves that propagate along the field). The gray curve corresponds to the case where the sound speed in the non-magnetic model is replaced by the fast-mode speed from the magnetic model (i.e., $c^{2} \rightarrow c_{f}^{2}=c^{2}+a^{2}$ ).

are then imposed at large height and large depth that ensure wave-like disturbances propagate out of the domain and evanescent modes decay. The central layer of the background model can have a general (tabulated) stratification. The models used in this investigation are based on model S (Christensen-Dalsgaard et al., 1996), with the gas pressure reduced to account for the magnetic pressure below the photosphere as in Crouch et al. (2005). The momentum equation,

$$
\rho_{0} \frac{\partial^{2} \xi}{\partial t^{2}}=-\nabla p_{1}+\frac{1}{\mu_{B}}\left(\nabla \times \mathbf{B}_{1}\right) \times \mathbf{B}_{0}-\rho_{1} g \hat{\mathbf{e}}_{z}+\rho_{0} g F_{z}(x, y, z, t) \hat{\mathbf{e}}_{z}-\Gamma(\mathbf{k}, \omega, z) \rho_{0} \frac{\partial \xi}{\partial t},
$$

where the subscript 0 refers to background quantities, subscript 1 is for Eulerian perturbations, includes finite-sized forcing, $F_{z}$, and damping, $\Gamma$, operators that peak near the photosphere (to simulate the influence of granulation on the waves). Examples of the power spectra produced by these models are shown in Figure 1. Although these models are simple in many respects, travel-time measurements taken from synthetic data produced by these models show qualitative similarities to measurements from sunspot-like magnetoconvection simulations and observational data of sunspots (Braun et al., 2010).

\section{A simple model for the wave-speed perturbation in sunspots}

We have tested the possibility that a simple model for the wave interactions with a sunspot can be constructed by replacing the sound speed in a non-magnetic model with the fast-mode speed, $c_{f}^{2}=a^{2}+c^{2}$, from a magnetic model. If this worked it would greatly simplify helioseismic inversions for subsurface structure below sunspots. In order to determine whether this is a viable approach we performed a series of tests using the TIM. Power spectra produced by this simple model are also shown in Figure 1. These preliminary results indicate that this simplified approach is not useful for modeling the wave-speed perturbation caused by a sunspot because the location of the peaks in the power and the corresponding linewidths do not agree with those for the magnetic model. 

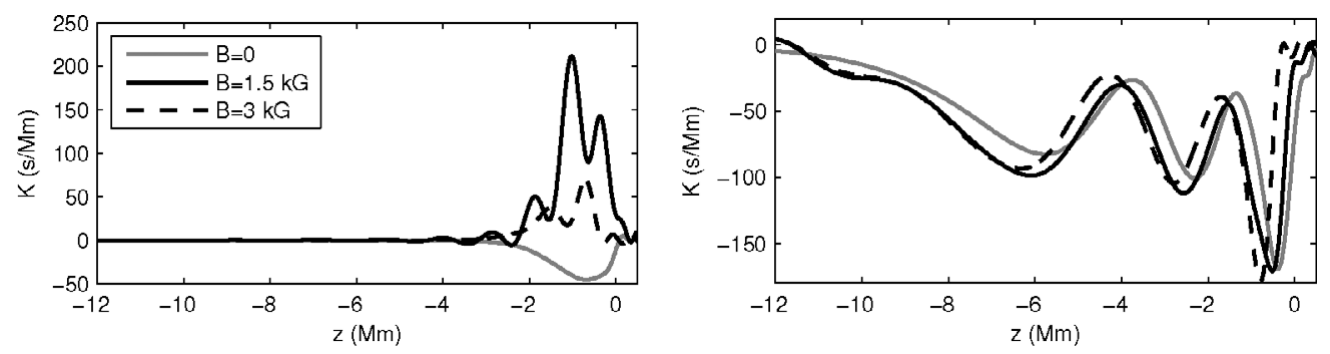

Figure 2. Time-distance travel-time sensitivity kernels for sound-speed perturbations as a function of depth for three different reference models: $B=0$ (gray curve), $B=1.5 \mathrm{kG}$ (black solid), and $B=3 \mathrm{kG}$ (black dashed). Left: phase-speed filter centered on $12.8 \mathrm{~km} / \mathrm{s}$ (TD1, Gizon \& Birch, 2005). Right: phase-speed filter centered on $35.5 \mathrm{~km} / \mathrm{s}$ (TD5). A vertical magnetic field clearly alters the kernels. In some cases (generally larger phase speed, e.g., TD5) the depthvariation is similar for the non-magnetic and magnetic cases, although the magnitude of the sensitivity is different. In other cases the effect of the field is drastic - both the sign and the depth-variation of the kernels is completely different in the non-magnetic and strong magnetic cases

\section{Inversions for sound speed in the presence of a magnetic field}

We now present an approach for inferring the sound speed in magnetic regions that includes the effects of the magnetic field on both the background structure and the wave mechanics. To construct sensitivity kernels for sound-speed perturbations we use a set of known, localized sound-speed perturbations based on B-splines. To produce a perturbation in $c^{2}$ we perturb the adiabatic index, $\Gamma_{1}$, keeping the pressure and density fixed. For each localized perturbation we generate power spectra with the TIM and then compute the time-distance correlation (filtering in phase speed and selecting different frequency ranges). From the correlation we compute travel times (using the method of Gizon \& Birch, 2002) and the travel-time shifts between each perturbed model and the (unperturbed) reference model. With the set of known sound-speed perturbations and corresponding travel-time shifts we can then compute kernels (see e.g., Fig. 2).

To infer the subsurface sound-speed profile we use regularized least-squares, which involves the minimization of

$$
\chi^{2}=\sum_{i} \frac{1}{\sigma_{i}^{2}}\left[\delta \tau_{i}-\int K_{i}(z) \frac{\delta c^{2}}{c^{2}}(z) d z\right]^{2}+\lambda \int\left|\frac{\delta c^{2}}{c^{2}}(z)\right|^{2} d z
$$

where $\delta \tau_{i}$ are the measured travel-time shifts, $\sigma_{i}$ are the measurement errors, $K_{i}$ are the sensitivity kernels for sound-speed perturbations $\delta c^{2} / c^{2}$, and $\lambda$ is the regularization parameter. We perform several "hare and hounds" tests using the translation-invariant model both to compute sensitivity kernels $K_{i}$ (for the "hounds") and to generate synthetic measurements (for the "hares"); results for sound-speed inversions in a background model with $3 \mathrm{kG}$ vertical field are presented in Figure 3. For this approach to produce reliable results we find that the travel-time shifts must be measured relative to a magnetic reference model (also with $3 \mathrm{kG}$ vertical field); travel-time shifts induced by a magnetic model measured relative to unperturbed quiet-Sun tend to be very large and hence outside the range of validity for linear inversions.

In the left-hand panel of Figure 3 we test the inversion algorithm using kernels for a non-magnetic background model; we emphasize that this approach is not formally consistent, however, if it worked it would be advantageous because the construction of kernels for a three-dimensional, magnetic sunspot model is computationally expensive. 

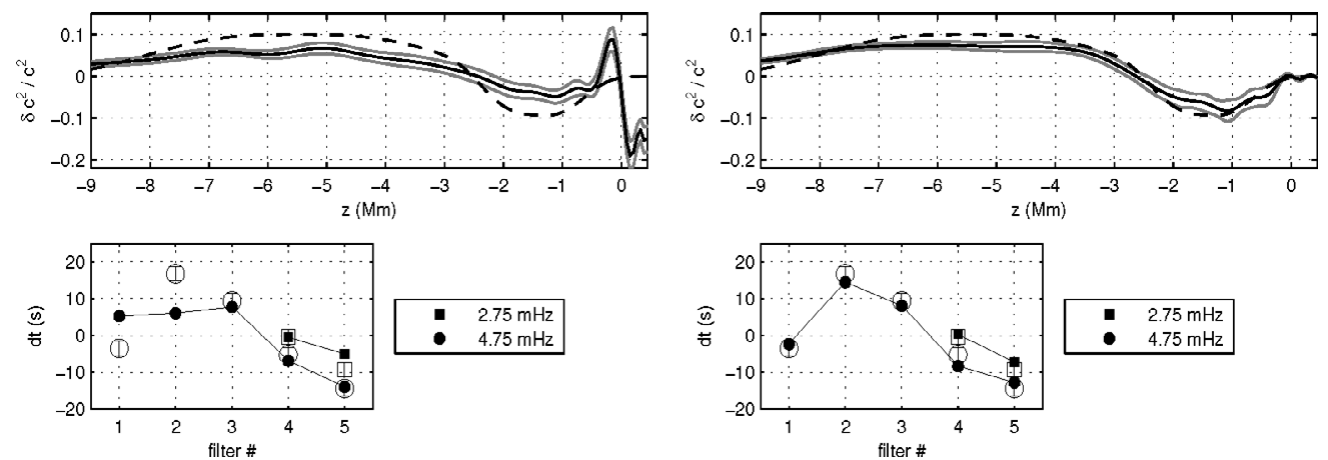

Figure 3. A "hare and hounds" test of inversions for a sound-speed perturbation in a background model with $3 \mathrm{kG}$ vertical magnetic field. Upper panels: the dashed curve is the sound-speed perturbation that was used to generate the synthetic measurements. The solid black curve is the perturbation retrieved from the inversion; the gray curves are the formal 1- $\sigma$ error estimates. Lower panels: travel-time shifts as a function of filter number for two representative frequency bands as labeled. The open symbols with error bars are the synthetic travel-time shifts and corresponding noise estimates. The closed symbols connected by lines are the travel-time shifts produced by the retrieved perturbation. Left: Inversion results using kernels for a non-magnetic background model. Right: Same as the left but using kernels for a background model with a $3 \mathrm{kG}$ vertical magnetic field.

We find that the results are dependent on the magnitude of the regularization parameter $\lambda$. After exploring a range of values we conclude that it is not possible to retrieve a solution using non-magnetic kernels that is both consistent with the travel-time measurements and has the expected sound-speed profile. In the right-hand panel of Figure 3 we test the self-consistent approach, using kernels constructed from simulations with a background model with a $3 \mathrm{kG}$ vertical field (matching the "hare"). These results show that when the correct kernels are employed this algorithm can retrieve the correct sound-speed profile.

\section{Conclusions}

We are developing inversion techniques for probing the subsurface structure and dynamics of sunspots and magnetic regions. This is challenging because the magnetic field in sunspots may affect both the background model (sound speed, density, etc.) and the wave mechanics (e.g., through mode conversion). We have evaluated a simple model for the wave-speed perturbation below sunspots that replaces the sound speed in a nonmagnetic model by the fast-mode speed, $c_{f}^{2}=a^{2}+c^{2}$, from a magnetic model; our results indicate that this approach is unlikely to be useful in modeling the wave-speed perturbations in sunspots. We develop and test an inversion algorithm for inferring the sound-speed perturbation in magnetic regions. Our preliminary results indicate that this algorithm is successful only when travel-time shifts are measured relative to a magnetic reference model (with a magnetic field that is close to that of the target of the inversion) and the models used to construct the sensitivity kernels properly account for the effects of the magnetic field on both the background model and the wave mechanics.

\section{Acknowledgement}

This work was supported by NASA contracts NNH09CE41C and NNG07EI51C. 


\section{References}

Braun, D. C., Birch, A. C., Crouch, A. D., \& Rempel, M. 2010, these proceedings

Cameron, R., Gizon, L., Schunker, H., \& Pietarila, A. 2010, Solar Phys., in press

Christensen-Dalsgaard, J., et al. 1996, Science, 272, 1286

Couvidat, S., Gizon, L., Birch, A. C., Larsen, R. M., \& Kosovichev, A. G. 2005, ApJS, 158, 217

Crouch, A. D. \& Cally, P. S. 2003, Solar Phys., 214, 201

Crouch, A. D. \& Cally, P. S. 2005, Solar Phys., 227, 1

Crouch, A. D., Cally, P. S., Charbonneau, P., Braun, D. C., \& Desjardins, M. 2005, MNRAS, 363, 1188

Cally, P. S. \& Goossens, M. 2008, Solar Phys., 251, 251

Fan, Y., Braun, D. C., \& Chou, D.-Y. 1995, Astrophys. J., 451, 877

Gizon, L. \& Birch, A. C. 2002, Astrophys. J., 571, 966

Gizon, L. \& Birch, A. C. 2005, Living Reviews in Solar Physics, 2, 6

Gizon, L., Birch, A. C., \& Spruit, H. C. 2010, ARA\&A, 48, 289

Hughes, S. J., Rajaguru, S. P., \& Thompson, M. J. 2005, Astrophys. J., 627, 1040

Kosovichev, A. G., Duvall, T. L., Jr., \& Scherrer, P. H. 2000, Solar Phys., 192, 159 